\title{
How strongly do hydrogen and water molecules stick to carbon nanomaterials?
}

Yasmine S. Al-Hamdani, Dario Alfè, and Angelos Michaelides

Citation: The Journal of Chemical Physics 146, 094701 (2017); doi: 10.1063/1.4977180

View online: http://dx.doi.org/10.1063/1.4977180

View Table of Contents: http://aip.scitation.org/toc/jcp/146/9

Published by the American Institute of Physics

\section{Articles you may be interested in}

The role of Stern layer in the interplay of dielectric saturation and ion steric effects for the capacitance of graphene in aqueous electrolytes

The Journal of Chemical Physics 146, 094101094101 (2017); 10.1063/1.4976991

A molecular dynamics investigation of the influence of water structure on ion conduction through a carbon nanotube

The Journal of Chemical Physics 146, 074502074502 (2017); 10.1063/1.4975690

Static polarizabilities and C6 dispersion coefficients using the algebraic-diagrammatic construction scheme for the complex polarization propagator

The Journal of Chemical Physics 146, 094301094301 (2017); 10.1063/1.4977039

Effects of translational and rotational degrees of freedom on properties of the Mercedes-Benz water model The Journal of Chemical Physics 146, 094505094505 (2017); 10.1063/1.4977214

Controlling the conductance of molecular junctions using proton transfer reactions: A theoretical model study The Journal of Chemical Physics 146, 092317092317 (2017); 10.1063/1.4974512

Comparing van der Waals DFT methods for water on $\mathrm{NaCl}(001)$ and $\mathrm{MgO}(001)$

The Journal of Chemical Physics 146, 064703064703 (2017); 10.1063/1.4971790

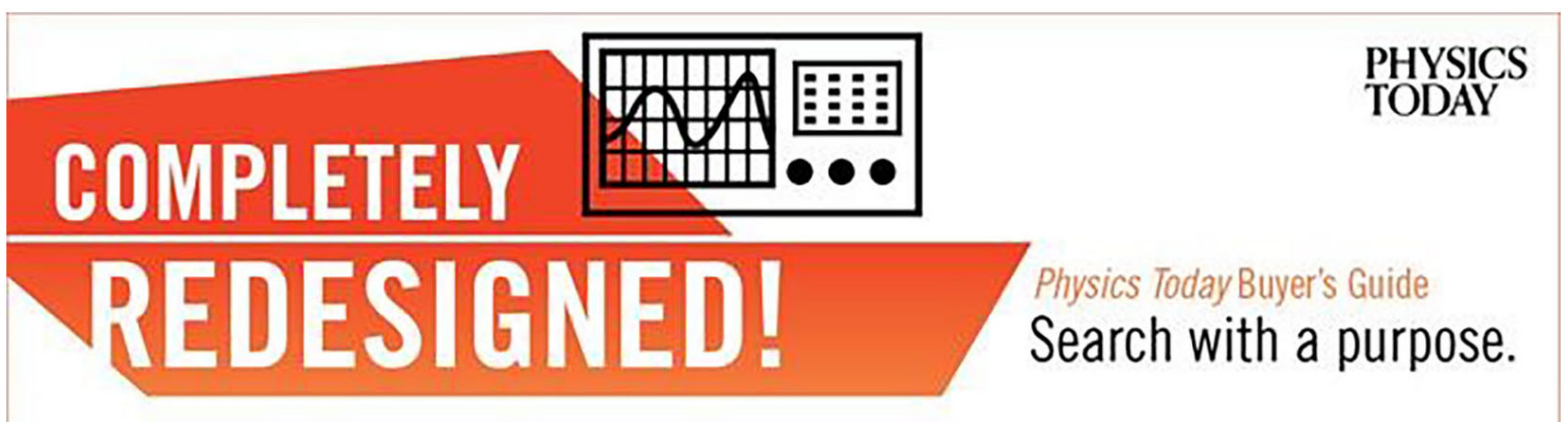




\title{
How strongly do hydrogen and water molecules stick to carbon nanomaterials?
}

\author{
Yasmine S. Al-Hamdani, ${ }^{1,2}$ Dario Alfè,,$^{1,3}$ and Angelos Michaelides ${ }^{1,4, a)}$ \\ ${ }^{1}$ Thomas Young Centre and London Centre for Nanotechnology, 17-19 Gordon Street, \\ London WC1H OAH, United Kingdom \\ ${ }^{2}$ Department of Chemistry, University College London, 20 Gordon Street, London WC1H 0AJ, United Kingdom \\ ${ }^{3}$ Department of Earth Sciences, University College London, Gower Street, London WC1E 6BT, United Kingdom \\ ${ }^{4}$ Department of Physics and Astronomy, University College London, Gower Street, London WC1E 6BT, \\ United Kingdom
}

(Received 29 November 2016; accepted 10 February 2017; published online 2 March 2017)

\begin{abstract}
The interaction strength of molecular hydrogen and water to carbon nanomaterials is relevant to, among many applications, hydrogen storage, water treatment, and water flow. However, accurate interaction energies for hydrogen and water with carbon nanotubes (CNTs) remain scarce despite the importance of having reliable benchmark data to inform experiments and to validate computational models. Here, benchmark fixed-node diffusion Monte Carlo (DMC) interaction energies are provided for hydrogen and water monomers inside and outside a typical zigzag CNT. The DMC interaction energies provide valuable insight into molecular interactions with CNTs in general and are also expected to be particularly relevant to gas uptake studies on CNTs. In addition, a selection of density functional theory (DFT) exchange-correlation $(x c)$ functionals and force field potentials that ought to be suitable for these systems is compared. An unexpected variation is found in the performance of DFT van der Waals (vdW) models in particular. An analysis of the peculiar discrepancy between different $\mathrm{vdW}$ models indicates that medium-range correlation (at circa 3 to $5 \AA$ ) plays a key role inside CNTs and is poorly predicted by some $\mathrm{vdW}$ models. Using accurate reference information, this work reveals which $x c$ functionals and force fields perform well for molecules interacting with CNTs. The findings will be valuable to future work on these and related systems that involve molecules interacting with low-dimensional systems. Published by AIP Publishing. [http://dx.doi.org/10.1063/1.4977180]
\end{abstract}

\section{INTRODUCTION}

Carbon nanotubes (CNTs) have been found to facilitate ultra-fast diffusion, ${ }^{1-8}$ desalination, ${ }^{9,10}$ and water treatment, ${ }^{11}$ and are also being developed into biochemical sensors. ${ }^{12-17}$ The binding strength of molecules to carbon nanomaterials is particularly important because it impacts macroscopic properties such as the contact angle, slip length, and gas storage capacity of nanomaterials, see, e.g., Refs. 10 and 18-22. However, despite the thousands of papers on CNTs, the binding energy of a single $\mathrm{H}_{2}$ or water molecule on a CNT is still not well established. ${ }^{23-26}$

Obtaining well defined experimental adsorption energies has always been a challenge, ${ }^{27,28}$ particularly so here, because of the difficulties in studying clean surfaces of carbon nanomaterials and pure bundles of CNTs experimentally. For instance, in the absence of benchmark adsorption energies, $\mathrm{H}_{2}$ was initially thought to adsorb on CNTs by as much as $\sim 200 \mathrm{meV}$ and thus, CNTs were stipulated to be viable materials for storing $\mathrm{H}_{2}$ gas. ${ }^{24,29}$ However, more recent estimates of the $\mathrm{H}_{2}$ adsorption energy on CNTs suggest that it is considerably lower $(\sim 50 \mathrm{meV}))^{30,31}$ Correspondingly, the experimentally reported $\mathrm{H}_{2}$ storage capacity of CNTs has decreased in the past 20 years, falling from $14 \mathrm{wt} . \%$ to around $2 \mathrm{wt} . \%{ }^{24}$

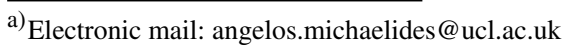

Adsorption energies can be predicted from theory and this is particularly useful given the scarcity of experimental reference binding energies. However, it is essential to capture the weak dispersion interactions that are prevalent in physisorption systems. Modelling these interactions accurately is a formidable challenge, especially in extended low dimensional systems $^{32-38}$ where the size of the system can pose an additional challenge. Since various macroscopic properties hinge on the atomic-scale interactions, even a small deviation in the adsorption energy can change the predicted behavior of a system. For example, Hummer et al. have shown that a range of adsorption energies and very small changes in the water-carbon interaction can impact upon whether water enters a CNT or not. ${ }^{39}$ Therefore, it is important to have accurate underlying models that provide reliable predictions.

The majority of computational studies focusing on either $\mathrm{H}_{2}$ or water on carbon nanomaterials use classical force fields with Lennard-Jones (LJ) type potentials to model the intermolecular interactions (see, e.g., Refs. 2, 19, 39, and 40). Density functional theory (DFT) is also seeing increasing application for such systems (see, e.g., Refs. 18 and 41-49). However, the predicted adsorption energies differ from one force field model to the next, and the same is true for different DFT exchange-correlation $(x c)$ functionals. It is not clear which of these methods gives more accurate results and only tentative comparisons can be made from the literature, since different types of CNTs and adsorbate configurations have 
been reported on. A number of force field studies have relied on experimental adsorption energies of $\mathrm{H}_{2}{ }^{40}$ and the contact angle of water on graphite ${ }^{50}$ - a material which is physically different to either graphene or CNTs. However, Leroy et al. have shown that the ability to reproduce the correct adsorption energy between water and the substrate in a force field leads to more accurate results, ${ }^{51}$ and therefore accurate reference information is needed.

There are a number of ways to compute accurate adsorption energies and here, benchmark interaction energies are provided from fixed-node diffusion Monte Carlo (DMC) on an extended CNT for the first time. DMC is explicitly correlated and accounts for exact exchange, thus it is able to capture weak interactions that contain a significant proportion of van der Waals (vdW) forces. Previously, Lei et al. employed density fitted local coupled cluster with single, double, and perturbative triple excitations (DF-LCCSD(T)) to compute interaction energy curves for a water monomer with non-periodic, $\mathrm{H}$ capped segments of CNTs of varying curvature. ${ }^{25}$ This was an incredibly impressive study; however, long-range charge density fluctuations on the nanometre scale can impact upon the interactions of low-dimensional systems like graphene and CNTs. ${ }^{32,33}$ Accounting for these long-range interactions requires one to go beyond localized segments of such systems and instead, to use a periodic unit cell to model an extended CNT. To this end, we have computed the physisorption energy of both $\mathrm{H}_{2}$ and water inside and outside a CNT in a periodic unit cell using DMC. The DMC reference interaction energies provide insight into molecular adsorption on CNTs, and also indicate that the uptake of $\mathrm{H}_{2}$ and water is favored in the sub-nanometre CNT considered here. We also compare the interaction energies with a graphene substrate and draw similarities with adsorption on the exterior of the CNT. In addition, direct comparison is made with some new and some widely used $x c$ functionals and force fields. We find that a particular class of $\mathrm{vdW} x c$ functionals overestimate the interaction energy inside the CNT by up to twice as much. This peculiar finding is considered more carefully, leading to some important implications for molecular adsorption inside CNTs and vdW methods.

\section{METHODS}

The DFT calculations were performed with VASP 5.4.1 $1^{52-55}$ with projector augmented wave (PAW) potentials. ${ }^{56,57}$ There are countless $x c$ functionals available in DFT $^{58-60}$ and it would not be feasible to test all of them, hence only a few widely used or relatively new $x c$ functionals have been chosen as part of this study. The various $x c$ functionals tested include the LDA, ${ }^{61} \mathrm{PBE},{ }^{62}$ dispersion corrected PBE (D2, ${ }^{63}$ D3, ${ }^{64,65}$ TSscs, ${ }^{66-68}$ and $\mathrm{MBD}^{67,69,70}$ ), and vdWinclusive functionals (original vdW-DF, ${ }^{71,72}$ optPBE-vdW, ${ }^{73}$ optB88-vdW, ${ }^{73,74}$ optB86b-vdW, ${ }^{75,76}$ vdW-DF2, ${ }^{77}$ rev-vdW$\left.\mathrm{DF} 2^{78}\right)$. In the case of the D3 correction, this is computed with the Becke and Johnson (BJ) damping function ${ }^{79-81}$ and with three-body Axilrod Teller contributions included. The revised version of the Vydrov and Van Voorhis non-local correlation functional, rVV10, ${ }^{82,83}$ is also included using Quantum Espresso. ${ }^{84}$ We have also tested the more recently developed strongly constrained and appropriately normalized (SCAN) functional of Sun et al. ${ }^{85}$ This functional is expected to outperform PBE for weakly interacting systems because it contains some non-local correlation from constraints based on the non-bonded interaction of an Ar dimer.

CNTs can vary in diameter and can be either metallic or semiconducting depending on their structure. The modelled CNT is $(10,0)$ in configuration, with a diameter of $7.8 \AA$, and belongs to the class of non-metallic zigzag CNTs. A CNT unit cell containing 80 carbon atoms was relaxed using a high $600 \mathrm{eV}$ energy cut-off as prescribed in VASP and PBE, PBE+TSscs, and vdW-DF2 functionals; the resulting cell parameters differ by $0.7 \%$ at most. PBE+TSscs predicted the nearest $\mathrm{C}-\mathrm{C}$ bond length to the experimental $\mathrm{C}-\mathrm{C}$ bond length in graphite $\left(1.421 \AA\right.$ ) at low temperatures ${ }^{86}$ and hence, the $8.58 \AA$ unit cell length predicted by this functional along the CNT axis was chosen for all further calculations. A unit cell length of $25 \AA$ was used along the other axes which allows for at least $\sim 17 \AA$ separation between periodic images of the CNT. The interaction energy of water/CNT was tested against a larger CNT unit cell of $12.8 \AA$ length at the DFT level. The difference in interaction energies was less than $3 \mathrm{meV}$ indicating that the water is well separated from its images. Water interaction energies were tested up to $10 \times 1 \times 1 \mathbf{k}$-points and convergence was reached already with just the $\Gamma$-point (within $2 \mathrm{meV}$ ) and subsequently used.

Graphene is a semi-metal for which a $(5 \times 5)$ unit cell was used with a $15 \AA$ long vacuum between graphene sheets. Following a convergence test on the number of k-points, a $4 \times 4 \times 1$ k-point mesh was chosen. After careful convergence tests for water/CNT and water/graphene interaction energies, a plane-wave energy cut-off of $500 \mathrm{eV}$ was applied for both systems. Hard PAWs with $700 \mathrm{eV}$ cut-off energy were also used to check convergence and standard PAWs were converged to less than $0.2 \mathrm{meV}$ for the interaction energy of water on graphene.

The lowest energy geometries of water interacting with CNTs are not entirely consistent in previous studies (varying by about $0.4 \AA$ ) which have mainly employed the LDA and PBE. ${ }^{41,42,87}$ Here, vdW-DF2 and PBE+TSscs were used to relax several starting configurations of water and $\mathrm{H}_{2}$, inside and outside the CNT, and on different sites above graphene. The lowest energy orientations were found to be consistent between PBE+TSscs and vdW-DF2 indicating that the choice of $x c$ functional does not have a great impact on the adsorbate geometry and vdW-DF2 relaxed structures were chosen for subsequent DFT, force field, and DMC calculations (see Fig. 1). In general, the potential energy surface is fairly smooth for graphene and even more so for the CNT, and as such, we expect small variations in the interaction energies for different configurations with other methods.

Force field calculations were performed using LAMMPS $^{88}$ with the TIP5P ${ }^{89}$ and $\mathrm{SPC} / \mathrm{E}^{90}$ models of water and $\mathrm{LCBOP}^{91}$ for the carbon substrates. The often used Werder potential ${ }^{50}$ for carbon-water interaction was tested along with recent carbonwater LJ type potentials that were fit to coupled cluster data for water on graphene ${ }^{92}$ and water on a H-capped segment of CNT. $^{25}$ 

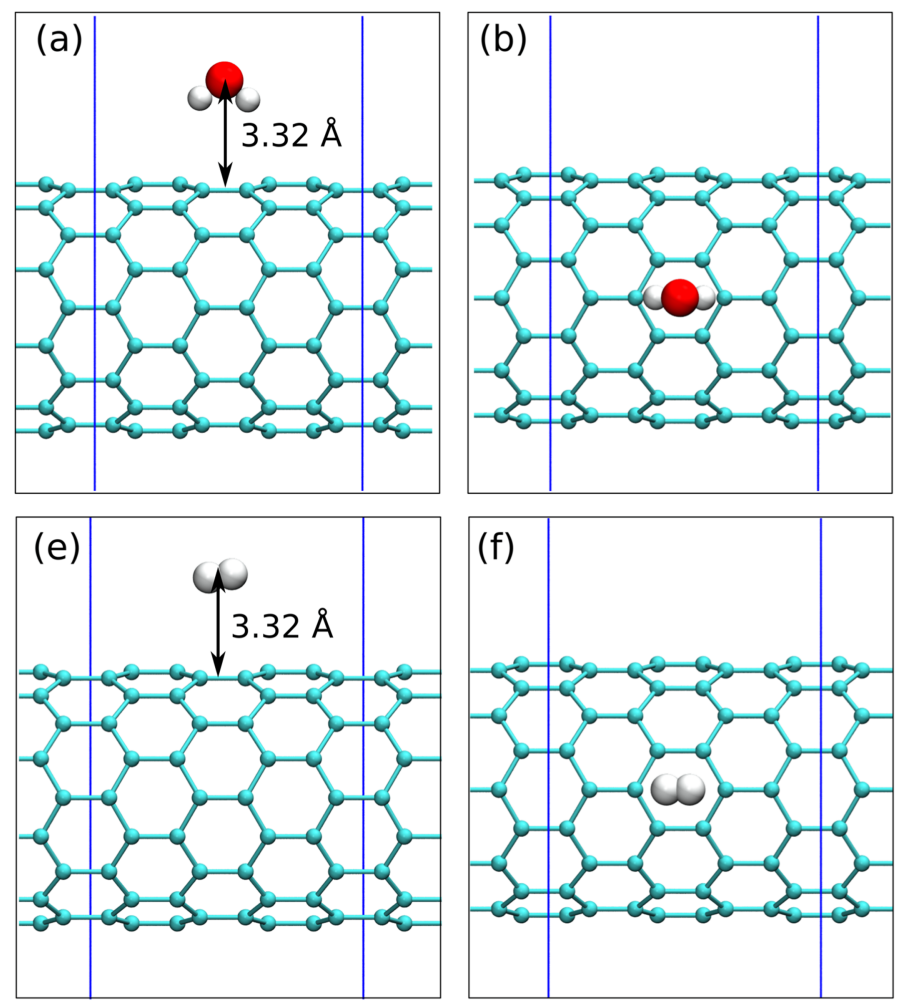

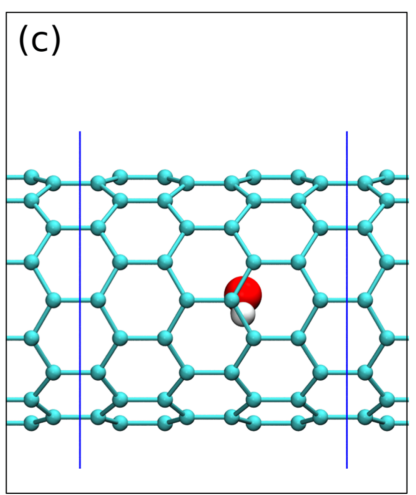

(d)

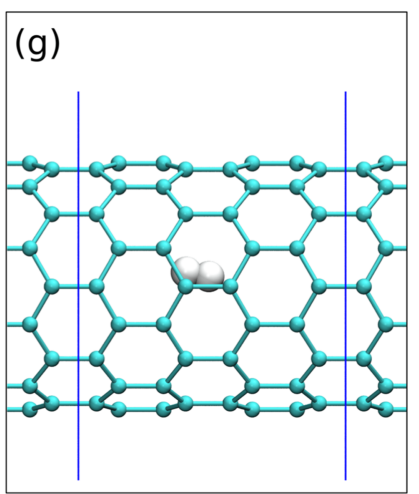

(h)
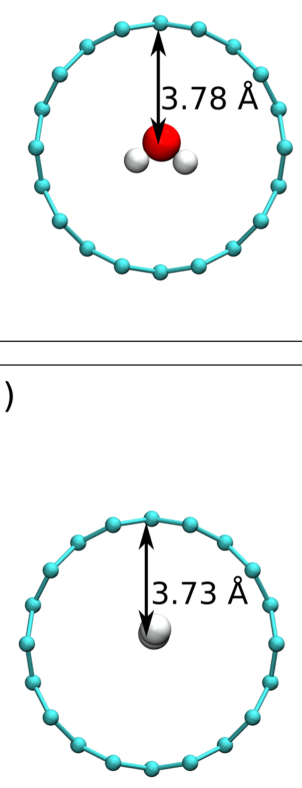

FIG. 1. Top panel: Unit cell of water outside ((a) and (b)) and inside ((c) and (d)) a CNT(10,0). Bottom panel: Unit cell of $\mathrm{H}_{2}$ outside ((e) and (f)) and inside ((g) and (h)) a CNT $(10,0)$. The unit cell is bounded by blue lines and contains 80 carbon atoms with a CNT diameter of $7.9 \AA$ A Configurations have been obtained from DFT geometry relaxations with the vdW-DF2 functional.

DMC calculations for CNT systems were performed using the CASINO code ${ }^{93}$ with the same cells and configurations as for the DFT calculations. A similar approach to previous benchmark DMC studies $34,94,95$ was used here. A plane-wave energy cut-off of $6800 \mathrm{eV}$ was applied to the LDA calculation of the trial wavefunctions in Quantum Espresso ${ }^{84}$ using the Trail and Needs pseudopotentials ${ }^{96,97}$ for all atoms. The resulting wavefunctions were expanded in terms of B-splines ${ }^{98}$ using a grid spacing, $a=\pi / G_{\max }$, where $G_{\max }$ is the plane wave cutoff wavevector. A Jastrow factor with up to three-body contributions was used to account for correlation and optimised using variational Monte Carlo. 1-D periodicity was applied along the CNT axis. For water/CNT systems, a time step of 0.015 a.u. was used in DMC whilst employing the locality approximation ${ }^{99}$ and 80000 walkers for each configuration. The DMC calculations were run until a stochastic error of 10-15 meV was reached, producing a combined error of less than $20 \mathrm{meV}$ in each interaction energy. A new implementation of $\mathrm{CASINO}^{100}$ has been used for $\mathrm{H}_{2}$ on CNT and graphene, which allows a larger time step ( 0.025 a.u.) to be used. A $3 \times 3$ unit cell of graphene was found to be large enough to avoid any interaction between the periodic images of $\mathrm{H}_{2}$ molecules at the DFT level. In addition, $\mathbf{k}$-point convergence was reached with $2 \mathbf{k}$-points at the DFT level. The total energy at each $\mathbf{k}$ point has equal weight in the total energy computed using 2 k-points. Trial wavefunctions were produced at each $\mathbf{k}$-point using Quantum Espresso, as prescribed for the CNT systems. The resulting DMC energies at each $\mathbf{k}$-point were averaged to give a final interaction energy for $\mathrm{H}_{2}$ on graphene.

The interaction energy of either water or $\mathrm{H}_{2}$ on the carbon substrates is defined as, $E_{\text {int }}=E_{a d s}^{\text {tot }}-E_{\text {far }}^{\text {tot }}$, where $E_{a d s}^{\text {tot }}$ is the total energy of the molecule/substrate system in the interacting configurations shown in Fig. 1. $E_{\text {far }}^{\text {tot }}$ is the total energy of the molecule/substrate system with the components separated by $12 \AA$. By defining the interaction energy in this way, it has been shown that size-consistency is maintained in the DMC calculation and the time step bias is also slightly reduced. ${ }^{100}$ This definition of the interaction energy is used to report the DMC, DFT, and force field results.

\section{RESULTS}

\section{A. Establishing accurate interaction energies using DMC}

The interaction energy of water and $\mathrm{H}_{2}$ has been computed with DMC, a selection of $x c$ functionals, and a few different classical water-substrate force field models. Table I reports the interaction energies for water at the CNT and in Table II results for $\mathrm{H}_{2}$ adsorption are reported. The reference DMC results are discussed first, followed by the performance of the $x c$ functionals, and finally some comments on the force field results are presented.

Let us begin by discussing the DMC results for water and $\mathrm{H}_{2}$. DMC predicts that water physisorbs on the exterior of the CNT with an interaction energy of $-80( \pm 19) \mathrm{meV}$ and on the interior with an interaction energy of $-244( \pm 17) \mathrm{meV}$. The $\operatorname{DF}-L C C S D(T)$ water adsorption energies computed by Lei et al. for a H-capped CNT segment with similar diameter ${ }^{25}$ are within $\sim 20 \mathrm{meV}$ of the DMC reference energies reported here. Despite the many papers dedicated to CNTs, experimental adsorption energies for water have not been reported ${ }^{50,92}$ 
TABLE I. Interaction energies (in $\mathrm{meV} / \mathrm{H}_{2} \mathrm{O}$ ) of water outside the CNT, inside the CNT, and on graphene. DMC energies are reported along with a selection of $x c$ functionals and force field models. Interaction energies that agree with DMC energies within the stochastic error are highlighted in bold.

\begin{tabular}{lccc}
\hline \hline Method & External-CNT & Internal-CNT & Graphene \\
\hline LDA $^{61}$ & -122 & $-\mathbf{2 3 7}$ & -124 \\
$\mathrm{PBE}^{62}$ & -26 & -84 & -21 \\
$\mathrm{SCAN}^{85}$ & $\mathbf{- 7 8}$ & -203 & -84 \\
\hline
\end{tabular}

Dispersion corrected $x c$ functionals

\begin{tabular}{|c|c|c|c|}
\hline $\mathrm{PBE}+\mathrm{D} 2^{62,63}$ & -120 & -305 & -136 \\
\hline $\mathrm{PBE}+\mathrm{D} 3^{62,64,65}$ & -113 & -293 & -126 \\
\hline PBE+TSscs $62,66,67$ & -137 & -327 & -158 \\
\hline $\mathrm{PBE}+\mathrm{MBD}^{62,69,108}$ & -99 & -293 & -130 \\
\hline $\mathrm{SCAN}+\mathrm{D} 3^{64,65,85,109}$ & -117 & -292 & -123 \\
\hline
\end{tabular}

Dispersion inclusive $x c$ functionals

\begin{tabular}{llll} 
Dispersion inclusive $x c$ functionals & & \\
vdW-DF $^{71,72}$ & -109 & -458 & -130 \\
optB88-vdW $^{75,76}$ & -123 & -457 & -152 \\
optPBE-vdW $^{73}$ & -137 & -506 & -169 \\
optB86b-vdW $^{75,76}$ & -122 & -459 & -154 \\
vdW-DF2 $^{77}$ & -108 & -397 & -129 \\
rev-vdW-DF2 $^{78}$ & $-\mathbf{9 7}$ & -365 & -119 \\
rVV10 $^{82,83}$ & -124 & -382 & -144 \\
\hline Force field methods $^{\text {Werder } \text { et al. }}{ }^{50}$ & & & \\
Lei et al. $^{25}$ & -50 & -179 & -63 \\
PHS $^{92}$ & -123 & -360 & -156 \\
\hline DMC & -99 & -304 & -125 \\
\hline \hline
\end{tabular}

$\overline{{ }^{\mathrm{a}} \text { The DMC interaction energy of water-graphene was calculated in a previous study by }}$ Ma et al. ${ }^{34}$

to the best of our knowledge. As a result, water-carbon potentials for modelling CNTs commonly rely on the water/graphite contact angle as a reference instead. ${ }^{50}$ Thus, theoretically computed adsorption energies of water/CNT serve as references for experimental as well as computational studies focusing on such systems.

In contrast to water, $\mathrm{H}_{2}$ physisorbs more weakly: $-26( \pm 10) \mathrm{meV}$ on the exterior of the CNT and $-115( \pm 11) \mathrm{meV}$ on the interior. Similar adsorption energies were obtained for $\mathrm{H}_{2}$ on a metallic CNT in a previous work by Rubeš and Bludskỳ using coupled cluster corrected DFT. ${ }^{26}$ Our results show that the $\mathrm{H}_{2}$ interaction is $\sim 50 \%$ weaker than water on each substrate, likely because of the stronger electrostatic interaction between the substrate and the permanent dipole of water. Interestingly, estimated $\mathrm{H}_{2}$ adsorption energies on CNTs from temperature programmed desorption (TPD) experiments are reported between 40 and $200 \mathrm{meV} \cdot{ }^{30,31,101-107}$ This large range has been attributed to different levels of purity of CNT bundles used in experiments, and possible interference from metal nanoparticles in the samples. ${ }^{103-105}$ Our DMC interaction energies suggest that $\mathrm{H}_{2}$ adsorption energies on pure CNTs should lie at the lower end of that range. The results also imply that considerably higher measurements of $\mathrm{H}_{2}$ adsorption energies indicate the presence of impurities or defects in CNTs.

The DMC interaction energy of water with graphene has previously been calculated to be $-70( \pm 10) \mathrm{meV}^{34}$ The
TABLE II. Interaction energies of $\mathrm{H}_{2}$ outside of the CNT, inside of the CNT, and on graphene in meV. A selection of $x c$ functionals and DMC energies is reported. Interaction energies that agree with DMC energies within the stochastic error are highlighted in bold.

\begin{tabular}{|c|c|c|c|}
\hline Method & External-CNT & Internal-CNT & Graphene \\
\hline $\mathrm{LDA}^{61}$ & -60 & -96 & -67 \\
\hline $\mathrm{PBE}^{62}$ & -6 & -22 & -5 \\
\hline $\operatorname{SCAN}^{85}$ & -17 & -50 & -22 \\
\hline \multicolumn{4}{|c|}{ Dispersion corrected $x c$ functionals } \\
\hline $\mathrm{PBE}+\mathrm{D} 2^{62,63}$ & -48 & $-\mathbf{1 1 7}$ & -59 \\
\hline $\mathrm{PBE}+\mathrm{D} 3^{62,64,65}$ & -52 & -128 & -53 \\
\hline PBE+TSscs ${ }^{62,66,67}$ & -60 & -138 & -72 \\
\hline $\mathrm{PBE}+\mathrm{MBD}^{62,69,108}$ & -39 & -107 & -53 \\
\hline $\mathrm{SCAN+D} 3^{64,65,85,109}$ & -38 & -100 & -43 \\
\hline \multicolumn{4}{|c|}{ Dispersion inclusive $x c$ functionals } \\
\hline vdW-DF ${ }^{71,72}$ & -59 & -230 & -77 \\
\hline optB88-vdW ${ }^{75,76}$ & -59 & -216 & -75 \\
\hline optPBE-vdW ${ }^{73}$ & -74 & -253 & -94 \\
\hline optB86b-vdW 75,76 & -58 & -221 & -79 \\
\hline vdW-DF2 ${ }^{77}$ & -55 & -181 & -69 \\
\hline rev-vdW-DF $2^{78}$ & -44 & -165 & -58 \\
\hline $\mathrm{rVV} 10^{82,83}$ & -52 & -151 & -65 \\
\hline DMC & $-26 \pm 10$ & $-115 \pm 11$ & $-24 \pm 11$ \\
\hline
\end{tabular}

interaction energy of $\mathrm{H}_{2}$ on graphene obtained here from DMC is $-24( \pm 11) \mathrm{meV}$. The interaction energies of water on graphene and the exterior of the CNT are very close in energy (within stochastic error). Likewise, the DMC interaction energies for $\mathrm{H}_{2}$ on the exterior of the $\mathrm{CNT}$ and on graphene are within stochastic error. The similar interaction energies on graphene and outside the CNT suggest that the curvature of this relatively small $(10,0)$ nanotube has at most a modest impact on the physisorption of small molecules on the exterior of the CNT. Experimentally produced CNTs can have much larger diameters than $\mathrm{CNT}(10,0),{ }^{1}$ so it is likely that interaction energies on those surfaces will be close to graphene.

Importantly, the DMC interaction energies inside the nanotube are three times larger than those obtained outside the nanotube. This relative difference between the interaction outside and inside of the nanotube will have a large impact on molecules entering a nanotube. ${ }^{39}$ As such, it will be another important aspect to consider when assessing the accuracy of various methods in Secs. III B-III D, starting with $x c$ functionals.

\section{B. Performance of $x c$ functionals: Challenge of internal interaction}

With the reference DMC information, we can assess the performance of a selection of $x c$ functionals listed in Tables I and II. We begin with the most commonly used functionals, the LDA and PBE. The LDA only accounts for short-range correlation and yet it overbinds both water and $\mathrm{H}_{2}$ outside the CNT by up to $30 \mathrm{meV}$, giving one of the worst performances for this configuration amongst the $x c$ functionals considered. On the other hand, the LDA prediction for water and $\mathrm{H}_{2}$ adsorption inside the CNT, -237 and $-96 \mathrm{meV}$, respectively, is in close agreement with DMC. This fortuitous performance of the LDA 


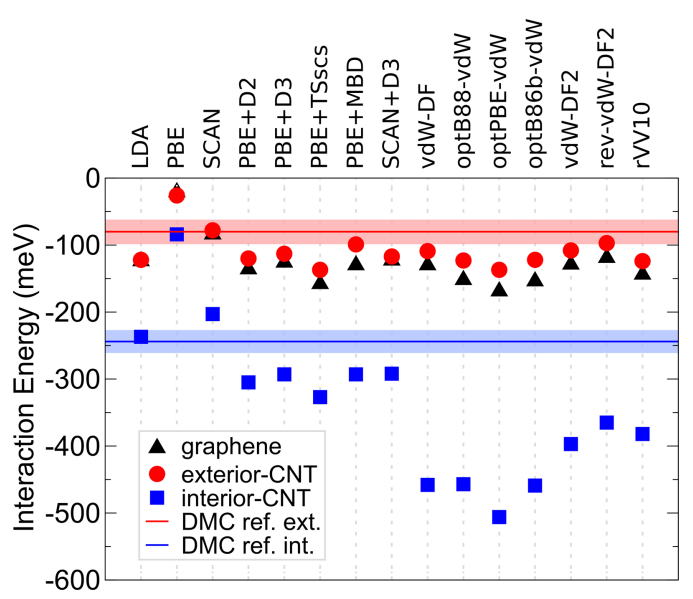

FIG. 2. Interaction energies of water inside (blue squares) and outside (red circles) the CNT with different $x c$ functionals and DMC. The DMC reference interaction energies are indicated by horizontal solid lines with the shaded area corresponding to the stochastic error. The interaction energy of water on graphene with different $x c$ functionals is also shown using black triangles. All energies are in $\mathrm{meV}$.

in physisorption systems is well-known $n^{60,94,110-112}$ and makes it difficult to draw physical insights from the LDA predictions. We can see from Fig. 2 that PBE severely underestimates the interaction energy of water on these low dimensional carbon substrates wherein dispersion is a significant part of the interaction. For $\mathrm{H}_{2}$ adsorption, PBE still underestimates the interaction energy of the interior configuration but appears to provide a closer estimate of the interaction energy for $\mathrm{H}_{2}$ outside of the CNT (see Fig. 3). The majority of previous DFT studies on graphene and CNTs have used PBE and the LDA to study water and $\mathrm{H}_{2} \cdot{ }^{41-45,47,48}$ The reported water-substrate and $\mathrm{H}_{2}$-substrate distances vary by up to $\sim 1 \AA$ in the literature and involve CNTs with different diameters and lengths. Even with these differences in mind, the interaction energies in previous studies are within $30 \mathrm{meV}$ of those reported here for LDA and PBE.

More promising performance is seen for the recently developed SCAN functional which predicts excellent physisorption

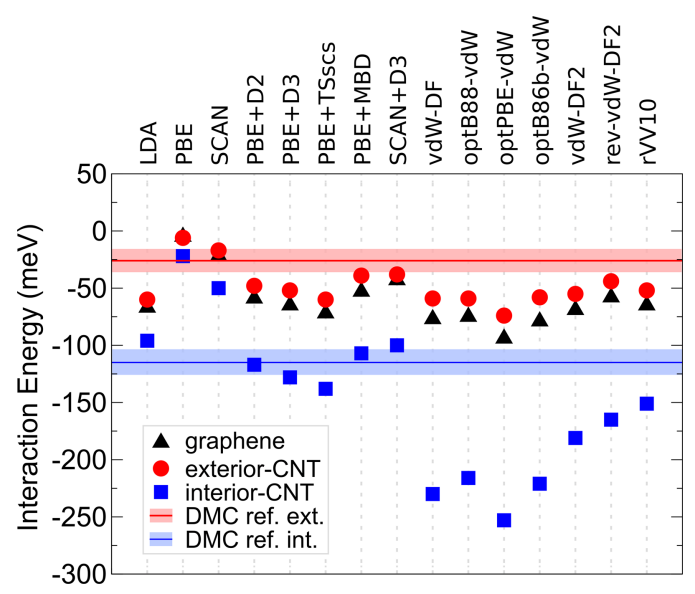

FIG. 3. Interaction energies of $\mathrm{H}_{2}$ inside (blue squares) and outside (red circles) the CNT with different $x c$ functionals and DMC. The DMC reference interaction energies are indicated by horizontal solid lines with the shaded area corresponding to the stochastic error. The interaction energy of $\mathrm{H}_{2}$ on graphene with different $x c$ functionals is also shown using black triangles. All energies are in $\mathrm{meV}$. energies for water $(-84 \mathrm{meV})$ and $\mathrm{H}_{2}(-17 \mathrm{meV})$ outside of the CNT. SCAN also predicts a similar physisorption energy of water on graphene to DMC (and RPA) from Ma et al. ${ }^{34}$ However, SCAN slightly underestimates the interaction energies by $\sim 30 \mathrm{meV}$ for both molecules inside the CNT, the underbinding results from the lack of dispersion energy are being taken into account.

There are two particularly common ways to account for dispersion interactions in DFT $x c$ functionals. The first is by adding a dispersion correction calculated from effective atomic dynamical polarizabilities and includes the D2, ${ }^{63} \mathrm{D} 3,{ }^{64,65}$ TSscs, ${ }^{66-68}$ and MBD ${ }^{67,69,70}$ methods. Hence, this class of $\mathrm{vdW}$ methods is referred to as dispersion corrected functionals. The second is based on the original vdW-DF from Dion et al. $^{71}$ in which two-body dispersion is calculated based on charge densities and is seamlessly incorporated in an $x c$ functional. This class of vdW methods is referred to as vdW-DFs or dispersion inclusive functionals. Some vdW methods have been shown to perform very well for weakly bound molecular systems (we refer the reader to the reviews in Refs. 32 and 113-115); though not as well for water adsorption on graphene ${ }^{34}$ and hexagonal boron nitride. ${ }^{94}$

For water/CNT and $\mathrm{H}_{2} / \mathrm{CNT}, \mathrm{PBE}+\mathrm{MBD}$ and $\mathrm{SCAN}+\mathrm{D} 3$ predict the best interaction energies amongst the vdW methods tested here for both exterior and interior adsorption. MBD takes into account beyond two-body correlation interactions and is therefore able to capture more effectively the dispersion that is present in the DMC reference interaction energies. In the systems considered here, $\mathrm{PBE}+\mathrm{MBD}$ predicts the largest contribution from beyond two-body correlation interactions for water inside the CNT, where it is $+26 \mathrm{meV}$. Most of this interaction energy arises from three-body interactions. Similarly, the D3 correction includes up to three-body correlation interactions and as we can see from Figs. 2 and 3 it also performs well. The three-body correlation interaction predicted by the $\mathrm{D} 3$ correction is $+34 \mathrm{meV}$ for water inside the CNT, in close agreement with the MBD correction. The performance of these $x c$ functionals is followed closely by the other dispersion corrected PBE functionals.

For exterior adsorption of water and $\mathrm{H}_{2}$ on the CNT, the vdW-DFs perform similarly to the dispersion correction approaches: over-binding by 20 to $40 \mathrm{meV}$ compared to DMC. The exceptions are vdW-DF2 and rev-vdW-DF2 which predict water interaction energies of -108 and $-97 \mathrm{meV}$, respectively. Rather strikingly, the vdW-DFs predict significantly more pronounced interaction energies inside the CNT, with up to a $250 \mathrm{meV}$ overestimation by optPBE-vdW. That is twice the DMC physisorption energy for water inside the CNT. In fact we see two regimes emerge for $\mathrm{vdW}$ functionals based on internal interaction energies from Figs. 2 and 3. Such a stark difference in the behavior of dispersion corrected DFT $x c$ functionals and vdW-DFs is not often seen in other systems and raises several questions which we address in Sec. III C. However, it is worth noting that all of the $x c$ functionals considered here correctly predict that water adsorption is about twice as strong as $\mathrm{H}_{2}$ adsorption. Therefore, DFT $x c$ functionals are likely to be useful methods for predicting the selectivity amongst molecules for adsorption on CNTs. Moreover, all of the $x c$ functionals with the exception of the LDA correctly predict a 
circa threefold increase in the adsorption energy of molecules from outside the CNT to inside the CNT.

\section{Understanding the performance of DFT: The importance of medium-range correlation}

The DFT results in this study indicate that molecular adsorption on CNTs is more accurately described by dispersion corrected $x c$ functionals as opposed to including vdW interactions in a seamless, though still approximate, manner. This is a somewhat unexpected finding because such a clear-cut difference in interaction energies between these two types of vdW functionals has not been observed previously. The reader is referred to some notable reviews, for example, Refs. 113-115, wherein various vdW-DFs and dispersion corrected functionals have been benchmarked on a number of weakly interacting systems, including the S22 data set and $\mathrm{H}_{2}$ adsorption on metal surfaces. In addition, various assumptions made in developing these $\mathrm{vdW}$ functionals are common to both types, and here we attempt to tease out the source of the disagreement.

For vdW-inclusive functionals the charge density is immediately brought into question since the dispersion contribution is calculated using the densities. To address this possibility, the vdW-DF interaction energy was calculated using the more localized Hartree-Fock density of the water/CNT configurations. The reduction in the interaction energy for the interior configuration of water is a mere $11 \mathrm{meV}$, going from $-458 \mathrm{meV}$ to $-447 \mathrm{meV}$. Hence, any delocalization error that is present in the vdW-inclusive functionals is not enough to explain the 100-200 meV overestimation seen here. For completeness, we also tested PBE0 ${ }^{116,117}$ which is a hybrid functional with a fraction of exact exchange. When combined with the D3 dispersion correction, the resulting interaction energies of water inside and outside the CNT are only $5 \mathrm{meV}$ less than PBE+D3 energies. Having established, therefore, that exact exchange has very little influence on the interaction of water with the CNT, we can proceed by analysing the contribution from nonlocal correlation energy to the interaction energies. Note that we use the term non-local correlation energy interchangeably with dispersion energy, to mean the long-range correlation interaction between electrons.

Fig. 4 shows an energy decomposition of the total interaction energy of water inside and outside the CNT, for the PBE+D3, vdW-DF, and vdW-DF2 functionals. The interaction energy is decomposed into the contribution from non-local correlation energy, $E_{n l c}$, and all remaining components of the energy, $E_{\text {rest }}$. Evidently from Fig. 4 , the contribution from $E_{n l c}$ in the vdW-DFs is much larger than with the D3 correction. However, when water is outside the CNT, the larger $E_{n l c}$ in the vdW-DFs is compensated by a repulsive interaction from $E_{\text {rest }}$. As a result, the three functionals predict almost the same interaction energy for water outside the CNT. On the other hand, the contribution from $E_{\text {rest }}$ in the vdW-DFs is much the same inside the CNT as it is outside (compare the left and right panel of Fig. 4). Whereas, there is a threefold increase in $E_{n l c}$ from water outside the CNT to inside, and this increased attraction inside the CNT is clearly not compensated by $E_{\text {rest }}$ in the vdW-DFs. In other words, for molecules outside of the CNT, the overestimation of non-local correlation interaction

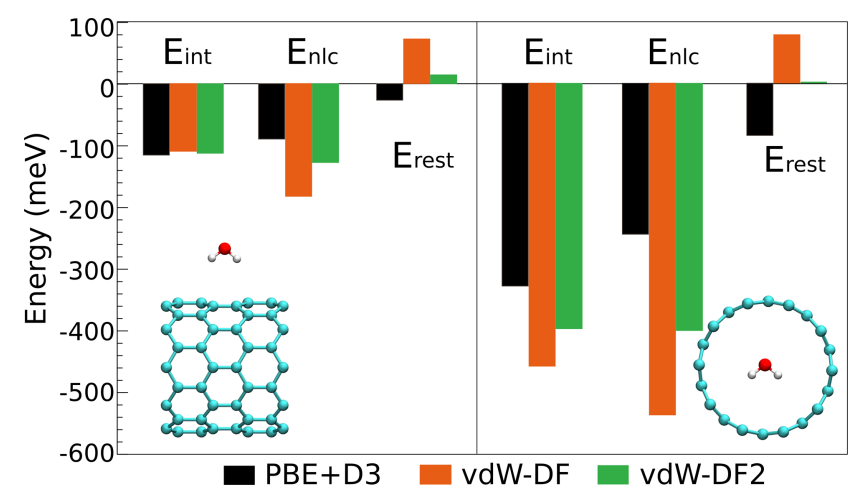

FIG. 4. Decomposition of the total interaction energy $\left(E_{\text {int }}\right)$ for PBE+D3, $\mathrm{vdW}-\mathrm{DF}$, and vdW-DF2. The contribution from non-local correlation energy $\left(E_{n l c}\right)$ and all other interactions that are collectively referred to as $E_{\text {rest }}$ is shown. $E_{\text {int }}=E_{\text {nlc }}+E_{\text {rest }}$.

by vdW-DFs is cancelled out by more repulsion in $E_{\text {rest }}$. This compensating effect is not present for molecules inside the CNT. The compensating effect in the dispersion inclusive functionals is present by design ${ }^{71,73,75,77}$ to help their accuracy on relatively small molecular dimers.

So why is water inside the CNT a particular problem for the dispersion inclusive methods? To answer this, we look more closely at the dispersion energy as a function of waterCNT distance for water outside the CNT, and compare this with the oxygen-carbon distances for water inside the CNT. This has been done by computing the interaction energy curve of water outside the CNT with PBE+D3, vdW-DF, and vdW$\mathrm{DF} 2$, and extracting the contribution from dispersion energy (i.e., $E_{n l c}$ ) at each point along the curves. The total interaction energy curves can be found in the supplementary material, but here we simply comment that the interaction energy curves for water outside the CNT are very similar to that of water on graphene.

In Fig. 5 the dispersion energy curves for water outside the CNT can be seen to vary significantly between the three functionals. As mentioned already, there is a pronounced repulsive interaction in the vdW-DFs that alleviates the large

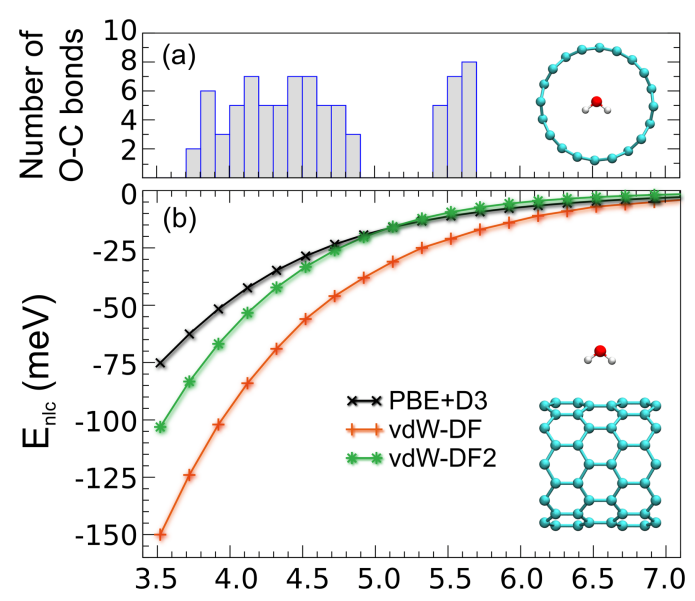

FIG. 5. (a) The bar chart shows the number of oxygen-carbon bonds at different bond lengths for water inside of the CNT. (b) The non-local correlation energy curves are plotted from PBE+D3, vdW-DF, and vdW-DF2, for water outside of the CNT: Single point calculations were used to compute the nonlocal correlation energy contribution $\left(E_{n l c}\right)$ to the interaction of water outside the CNT at a series of oxygen-CNT distances. 
non-local correlation energy for water outside the CNT, but crucially not for water inside the CNT. Comparing these dispersion energy curves with the frequency of oxygen-carbon bonds at a given distance for water inside the CNT in Fig. 5(a), we see that the majority of oxygen-carbon bonds inside the CNT lie within 3.5 to $5.0 \AA$. At these distances the dispersion energy is particularly large in the vdW-DFs compared to $\mathrm{PBE}+\mathrm{D} 3$. In the absence of an adequate repulsive interaction (as illustrated in Fig. 4), the total interactions at these medium-range distances are poorly described by the $\mathrm{vdW}$ inclusive functionals. This could be interpreted as too much correlation energy at medium-range distances or equally as not enough repulsive interaction to compensate for it. Note that this medium-range correlation regime refers to atomic separations larger than bonding distances (a few Ångstroms) and closer than the long-range limit where the interaction reaches the $1 / r^{6}$ limit $(\sim 10 \AA)$.

The reasonably good performance of dispersion corrected SCAN and PBE suggests that these describe the mediumrange interactions better. This is possibly due to the use of damping functions, ${ }^{65,118}$ that are used to adjust the short-range behavior of the dispersion correction with respect to the underlying $x c$ functional empirically. In this way, damping functions directly affect the medium-range interactions in the dispersion corrected functionals that we have tested.

Although we have not come across any studies showing or discussing two distinct regimes for the performance of vdW-DFs and dispersion corrected functionals, there are indications of this finding in previously computed interaction energy curves. In particular, the ordering of some $x c$ functionals at medium-range distances in the interaction energy curves of weakly interacting complexes in Refs. 94 and 119-123 closely match the order we see in Figs. 2 and 3.

The importance of medium-range correlation can also be seen by comparing the geometry optimized interaction energies for water inside and outside of CNTs with different diameters in Fig. 6. Water interaction energies outside the CNT show less than 7\% deviation between PBE+D3 and vdW-DF across all three CNT diameters, shown in Fig. 6. Whereas for water inside the CNT, the interaction energy difference between $\mathrm{PBE}+\mathrm{D} 3$ and vdW-DF increases rapidly from $9 \%$ to $30 \%$ as the CNT diameter decreases. For larger CNT

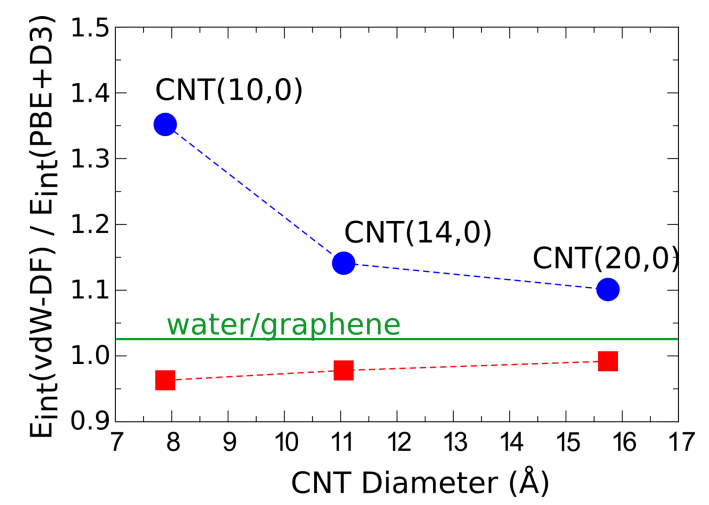

FIG. 6. Ratio of vdW-DF to PBE+D3 interaction energies for water inside (blue squares) and outside (red circles) of CNTs with increasing diameters: CNT(10,0), CNT(14,0), and CNT(20,0). The water/graphene ratio is indicated by the solid green line. diameters, there are fewer oxygen-carbon bonds at mediumrange distances for water inside the CNTs. The corresponding radial distribution functions between oxygen and carbon, $g_{O C}$, can be found in Fig. 2 of the supplementary material. This suggests that vdW-DF begins to overestimate the dispersion interactions more than $\mathrm{PBE}+\mathrm{D} 3$ for CNTs with diameters less than $\sim 10 \AA$. We expect this to be the case for all the other vdW-DFs tested in this paper, as well as the rVV10 functional.

\section{Reliable water-carbon potentials for water/CNT?}

The DMC simulations are also useful in helping to evaluate how standard force field models for the water-carbon interaction perform. Three LJ type force fields for the watercarbon substrate interaction have been tested here, referred to as Werder et al. ${ }^{50}$ Lei et al., ${ }^{25}$ and PHS (Pérez-Hernández and Schmidt). ${ }^{92}$ The potential by Werder et al. is one of the most commonly used for water/carbon systems and was designed to reproduce experimental water contact angles on graphite. ${ }^{50}$ In this potential only the $\mathrm{C}-\mathrm{O}$ interaction is defined $\left(\epsilon_{\mathrm{CO}}=4.549\right.$ meV and $\sigma_{\mathrm{CO}}=3.19 \AA$ ) and it was obtained by tuning $\epsilon_{\mathrm{CO}}$ until an experimental water/graphite contact angle was reproduced with the SPC/E model of water. It can be seen from Table I that this interaction potential leads to an underestimation in the interaction energy of water especially inside the CNT, where it is at least $40 \mathrm{meV}(20 \%)$.

Lei et al. have suggested a few different water-carbon potentials by manually fitting interaction parameters to DFLCCSD(T) interaction energy curves for water with H-capped segments of CNTs. It is recognised therein that water adsorption inside and outside the nanotube is not accurately predicted by any single set of parameters. We have chosen one that includes $\mathrm{C}-\mathrm{H}$ interaction parameters as well $\left(\epsilon_{\mathrm{CH}}=4.457 \mathrm{meV}\right.$ and $\left.\sigma_{\mathrm{CH}}=2.80 \AA\right) .{ }^{25}$ Using TIP5P for the water force field as prescribed, there is a threefold increase of the water interaction energy from the exterior $(-123 \mathrm{meV})$ to the interior $(-360 \mathrm{meV})$ of the $\mathrm{CNT}$, in agreement with the ratio from DMC. However, the interaction energies are overestimated outside (by $\sim 20 \%$ ) and inside the CNT (by $\sim 40 \%$ ). It is worth noting, however, that the orientation of water in the DF-LCCSD(T) calculations is different to the configuration studied here; with the $\mathrm{H}$ atoms of water parallel to the length of the CNT instead of perpendicular as shown in Fig. 1.

Another LJ type water-carbon potential based on the CCSD(T) water-graphene adsorption energy and the TIP5P model of water has been proposed by Pérez-Hernández and Schmidt. ${ }^{92}$ This PHS model was obtained by reproducing the CCSD(T) interaction energy of water in the up and down configurations on a 58 carbon atom segment of graphene. ${ }^{35}$ Orientation dependence is therefore built in by defining $\mathrm{C}-\mathrm{H}$ as well as $\mathrm{C}-\mathrm{O}$ interactions for water. From Table I, it can be seen that this potential performs very well, predicting $-99 \mathrm{meV}$ for water outside of the CNT, which is within the stochastic error of the DMC reference. In addition, for water inside the CNT the PHS force field performs as well as the dispersion corrected functionals (see Table I).

The sensitivity of the force fields to the form of parametrization is clear from the varying performance of the three force field models considered here. With the DMC 
reference interaction energies of water on the CNT, we can see that the PHS force field is particularly good for these systemsperforming on par with dispersion corrected $x c$ functionals. As demonstrated, the DMC reference interaction energies in this study could be used to determine the accuracy of future force field adsorption studies on these systems.

\section{DISCUSSION}

The benchmark DMC energies reported in this paper are the first, explicitly correlated and exact exchange, interaction energies for water and $\mathrm{H}_{2}$ on an extended CNT and are also intended to serve as references for other methods. Additional insight is given on the DMC results in this section and the significant overestimation by vdW-DFs for adsorption inside the CNT is addressed. We first comment on the appropriateness of the DMC method for these systems in the context of other benchmark methods, and we make an estimate of finite size effects in the DMC energies. Later, we comment on the findings in the context of other types of nanotubes, namely, metallic CNTs and insulating boron nitride nanotubes (BNNTs).

Let us first consider the suitability of DMC for interaction of molecules with the $\operatorname{CNT}(10,0)$ that is considered here. The DMC calculations in this study used a singledeterminant approach. This is expected to be sufficient since multi-reference character is unlikely given that the bandgap of CNT $(10,0)$ is $\sim 1 \mathrm{eV}$ even at the GGA level. ${ }^{124-126}$ Furthermore, an important and challenging feature of CNTs that needs to be accounted for is their extended and delocalized nature. To this end, DMC can be efficiently used with periodic boundary conditions and as a result, it is free of localization approximations in the charge density and polarizability. On the contrary, such approximations are inherent in non-periodic calculations using CCSD(T). Using unit cells with periodic boundary conditions, however, leads to finite size effects in DMC that merit further comment.

The main source of finite size effects relevant to the CNT studied here with DMC is the long-range nature of Coulomb interactions. Such long-range Coulomb interactions can extend to the nanometre scale ${ }^{33}$ and are prevalent in lowdimensional extended materials with small bandgaps. ${ }^{33,127}$ Capturing long-range interactions at the nanometre scale requires unit cells that extend to the same lengths as the interactions, i.e., a few nanometres. The unit cell used in this work contains 80 carbon atoms and is $8.58 \AA$ along the CNT axis. Although DMC provides a many-body solution for this relatively large system, larger unit cells become increasingly prohibitive. Instead, finite size effects can be estimated in the unit cell used here by invoking the MBD correction at the DFT level. Unlike DMC, the MBD correction is computationally inexpensive and can therefore be used in large unit cells to capture the contribution from long-range Coulomb interactions.

We find that the MBD correction to PBE is converged with a k-point mesh of $2 \times 1 \times 1$ which is equivalent to doubling the unit cell along the length of the CNT. The MBD correction increases the water interaction energy by $12 \mathrm{meV}$ outside the $\mathrm{CNT}$ and by $16 \mathrm{meV}$ inside the CNT. For the $\mathrm{H}_{2}$ interaction the MBD correction is $5-8 \mathrm{meV}$ only. These corrections are applicable to all explicitly correlated calculations with a periodic unit cell, including DMC, and should be taken as the finite size error corrections. Shifting the DMC reference energies in this manner increases the interactions, but since the corrections are relatively small they remain within the stochastic error bars of DMC.

Let us also consider the DFT results reported here in the context of previous predictions. Dobson et al. ${ }^{32,127}$ and Misquitta et al. ${ }^{128}$ have previously identified key assumptions in $\mathrm{vdW}$ approximations based on $1 / r^{6}$ behavior. They point out that such approximations (present in the dispersion corrections and vdW-DFs) render these methods incapable of accounting for non-additivity in polarizabilities, which are particularly relevant for extended low-dimensional systems such as CNTs. Nonetheless, DMC has been used to show in this study that dispersion corrected methods perform relatively well for molecular adsorption on a non-metallic CNT.

The neglect of non-additivity in polarizabilities is expected to be important in metallic or small bandgap systems. ${ }^{32,127,128}$ Therefore, it would be interesting to know if the performance of the dispersion corrected methods holds for nanotubes with different electronic properties. This would require more high-accuracy benchmark calculations on a metallic CNT and an insulating nanotube such as a BNNT. Although there are currently no DMC references for these systems, we computed the adsorption energy of water inside and outside a metallic $\operatorname{CNT}(6,6)$ and an insulating $\operatorname{BNNT}(10,0)$, with PBE+D3 and vdW-DF. These nanotubes have similar diameters to the $\mathrm{CNT}(10,0)$. Interestingly, we find that the same trends in energy are exhibited in these nanotubes as for water on $\mathrm{CNT}(10,0)$, regardless of the electronic properties of the nanotubes. The adsorption energy outside the $\mathrm{CNT}(6,6)$ is $\sim-12 \mathrm{meV}$ with PBE+D3 and vdW-DF, whereas inside the CNT $(6,6)$, vdW-DF predicts a much stronger adsorption energy $(-420 \mathrm{meV})$ compared to PBE+D3 (-304 meV). The interaction energies of water on $\operatorname{BNNT}(10,0)$ are 10-20 meV stronger than on the CNTs, but vdW-DF still predicts a considerably larger interaction energy for water inside the BNNT than PBE+D3. Thus, these calculations indicate that the conclusions made here about the performance of various $x c$ functionals likely apply to other systems.

\section{CONCLUSIONS}

Reference DMC interaction energies have been computed for water and $\mathrm{H}_{2}$ on the outside and inside of the zigzag $\mathrm{CNT}(10,0)$ and also for $\mathrm{H}_{2}$ on graphene. Adsorption of either water or $\mathrm{H}_{2}$ inside this nanotube is about three times larger than outside, suggesting that the uptake of water and $\mathrm{H}_{2}$ is possible in some sub-nanometre CNTs. With regard to the wide-ranging experimental adsorption energies reported for $\mathrm{H}_{2}$ on carbon nanomaterials, the DMC reference energies for $\mathrm{H}_{2}$ corroborate that the adsorption energy is weak at around $-100 \mathrm{meV}$ or less. In addition, the adsorption energy of water on the CNT is a factor of $\sim 2$ larger than $\mathrm{H}_{2}$ and thus, $\mathrm{H}_{2}$ is less likely to be adsorbed on a CNT in the presence of water.

Three water-carbon force fields were benchmarked against DMC, including the widely used Werder et al. potential. Naturally, the results are very sensitive to the parameters 
and underlying model, but we find that for water on $\operatorname{CNT}(10,0)$ the force field model given by Pérez-Hernández and Schmidt predicts interaction energies in good agreement with DMC. In contrast, a selection of widely used and new $x c$ functionals considered here is unable to accurately predict the interaction energies for these systems. Strikingly, there is a clear distinction between dispersion corrected $x c$ functionals-which only slightly overestimate the interaction energies-and dispersion inclusive functionals. The latter strongly over-bind molecules inside the CNT: up to twice as much. An analysis of DFT energies indicates that the inaccuracy arises from medium-range correlation, which seems to be poorly described by the dispersion inclusive functionals. These findings also hold for molecular adsorption inside a metallic CNT and a $\mathrm{BNNT}$, indicating that the error from medium-range correlation is wide-spread and likely to manifest in other systems. Indeed, benchmark studies of water on other low-dimensional materials suggest they too lack consistent accuracy. ${ }^{34,94}$

Finally, we expect that the reference adsorption energies of water and $\mathrm{H}_{2}$ on CNTs established in this work will help to understand and interpret studies regarding bio-sensing, storage capacities, slip lengths, and molecular transport in CNTs, among other applications.

\section{SUPPLEMENTARY MATERIAL}

See supplementary material for additional information regarding the interaction energy curve of water on graphene and outside $\operatorname{CNT}(10,0)$ and the radial distribution function for $\mathrm{O}-\mathrm{C}$ bonds in CNTs of different diameters. Details of the structures that have been used for the benchmarking can also be found therein.

\section{ACKNOWLEDGMENTS}

We are grateful for support from University College London and Argonne National Laboratory (ANL) through the Thomas Young Centre-ANL initiative. Some of the research leading to these results has received funding from the European Research Council under the European Union's Seventh Framework Programme (No. FP/2007-2013)/ERC Grant Agreement No. 616121 (HeteroIce project). A.M. is supported by the Royal Society through a Wolfson Research Merit Award. This research also used resources of the Argonne Leadership Computing Facility at Argonne National Laboratory, which is supported by the Office of Science of the U.S. DOE under Contract No. DE-AC02-06CH11357. This research also used resources as part of an INCITE project (awarded to D.A.) at the Oak Ridge National Laboratory (Rhea/Eos), which is supported by the Office of Science of the U.S. Department of Energy (DOE) under Contract No. DEAC05-00OR22725. In addition, we are grateful for the computing resources provided by the London Centre for Nanotechnology and Research Computing at University College London.

${ }^{1}$ J. K. Holt, Science 312, 1034 (2006).

${ }^{2}$ K. Falk, F. Sedlmeier, L. Joly, R. R. Netz, and L. Bocquet, Nano Lett. 10, 4067 (2010).

${ }^{3}$ E. Secchi, S. Marbach, A. Niguès, D. Stein, A. Siria, and L. Bocquet, Nature $\mathbf{5 3 7 ,} 210$ (2016).
${ }^{4}$ M. Whitby, L. Cagnon, M. Thanou, and N. Quirke, Nano Lett. 8, 2632 (2008).

${ }^{5}$ M. Majumder, N. Chopra, R. Andrews, and B. J. Hinds, Nature 438, 930 (2005).

${ }^{6}$ M. Ma, F. Grey, L. Shen, M. Urbakh, S. Wu, J. Z. Liu, Y. Liu, and Q. Zheng, Nat. Nanotechnol. 10, 692 (2015).

${ }^{7}$ J. Su and K. Yang, ChemPhysChem 16, 3488 (2015).

${ }^{8}$ A. Michaelides, Nature 537, 171 (2016).

${ }^{9}$ M. A. Tofighy, Y. Shirazi, T. Mohammadi, and A. Pak, Chem. Eng. J. 168, 1064 (2011).

${ }^{10}$ R. Das, M. E. Ali, S. B. A. Hamid, S. Ramakrishna, and Z. Z. Chowdhury, Desalination 336, 97 (2014).

${ }^{11}$ B. Lee, Y. Baek, M. Lee, D. H. Jeong, H. H. Lee, J. Yoon, and Y. H. Kim, Nat. Commun. 6, 7109 (2015).

${ }^{12}$ M. F. L. De Volder, S. H. Tawfick, R. H. Baughman, and A. J. Hart, Science 339, 535 (2013).

${ }^{13}$ D. R. Kauffman and A. Star, Angew. Chem., Int. Ed. 47, 6550 (2008).

${ }^{14}$ P. Qi, O. Vermesh, M. Grecu, A. Javey, Q. Wang, H. Dai, S. Peng, and K. J. Cho, Nano Lett. 3, 347 (2003).

${ }^{15}$ M. Dionisio, J. M. Schnorr, V. K. Michaelis, R. G. Griffin, T. M. Swager, and E. Dalcanale, J. Am. Chem. Soc. 134, 6540 (2012).

${ }^{16}$ S. F. Liu, S. Lin, and T. M. Swager, ACS Sens. 1, 354 (2016).

${ }^{17}$ Z. Zanolli, R. Leghrib, A. Felten, J. J. Pireaux, E. Llobet, and J. C. Charlier, ACS Nano 5, 4592 (2011).

${ }^{18}$ L. Mandeltort, D.-L. Chen, W. A. Saidi, J. K. Johnson, M. W. Cole, and J. T. Yates, J. Am. Chem. Soc. 135, 7768 (2013).

${ }^{19}$ A. Striolo, A. Michaelides, and L. Joly, Annu. Rev. Chem. Biomol. Eng. 7, 533 (2016).

${ }^{20}$ E. A. Müller, Curr. Opin. Chem. Eng. 2, 223 (2013).

${ }^{21}$ S. Guo, E. R. Meshot, T. Kuykendall, S. Cabrini, and F. Fornasiero, Adv. Mater. 27, 5726 (2015).

${ }^{22}$ L. Bocquet and E. Charlaix, Chem. Soc. Rev. 39, 1073 (2010).

${ }^{23}$ R. Ströbel, J. Garche, P. T. Moseley, L. Jörissen, and G. Wolf, J. Power Sources 159, 781 (2006).

${ }^{24}$ C. Liu, Y. Chen, C.-Z. Wu, S.-T. Xu, and H.-M. Cheng, Carbon 48, 452 (2010).

${ }^{25}$ S. Lei, B. Paulus, S. Li, and B. Schmidt, J. Comput. Chem. 37, 1313 (2016).

${ }^{26}$ M. Rubeš and O. Bludský, ChemPhysChem 10, 1868 (2009).

${ }^{27}$ C. T. Campbell and J. R. V. Sellers, Chem. Rev. 113, 4106 (2013).

${ }^{28}$ J. Wellendorff, T. L. Silbaugh, D. Garcia-Pintos, J. K. Nørskov, T. Bligaard, F. Studt, and C. T. Campbell, Surf. Sci. 640, 36 (2015).

${ }^{29}$ A. G. Klechikov, G. Mercier, P. Merino, S. Blanco, C. Merino, and A. V. Talyzin, Microporous Mesoporous Mater. 210, 46 (2015).

${ }^{30}$ A. Züttel, P. Sudan, P. Mauron, T. Kiyobayashi, C. Emmenegger, and L. Schlapbach, Int. J. Hydrogen Energy 27, 203 (2002).

${ }^{31}$ Y. Ye, C. C. Ahn, C. Witham, B. Fultz, J. Liu, A. G. Rinzler, D. Colbert, K. A. Smith, and R. E. Smalley, Appl. Phys. Lett. 74, 2307 (1999).

${ }^{32}$ J. F. Dobson and T. Gould, J. Phys.: Condens. Matter 24, 073201 (2012).

${ }^{33}$ A. Ambrosetti, N. Ferri, R. A. DiStasio, and A. Tkatchenko, Science 351, 1171 (2016).

${ }^{34}$ J. Ma, A. Michaelides, D. Alfè, L. Schimka, G. Kresse, and E. Wang, Phys. Rev. B 84, 033402 (2011)

${ }^{35}$ E. Voloshina, D. Usvyat, M. Schuz, Y. Dedkov, and B. Paulus, Phys. Chem. Chem. Phys. 13, 12041 (2011).

${ }^{36}$ G. R. Jenness, O. Karalti, and K. D. Jordan, Phys. Chem. Chem. Phys. 12, 6375 (2010).

${ }^{37}$ G. R. Jenness and K. D. Jordan, J. Phys. Chem. C 113, 10242 (2009).

${ }^{38}$ D. Feller and K. D. Jordan, J. Phys. Chem. A 104, 9971 (2000).

${ }^{39}$ G. Hummer, J. C. Rasaiah, and J. P. Noworyta, Nature 414, 188 (2001).

${ }^{40}$ A. S. Fedorov and A. F. Sadreev, Eur. Phys. J. B 69, 363 (2009).

${ }^{41}$ J. Zhao, A. Buldum, J. Han, and J. P. Lu, Nanotechnology 13, 195 (2002).

${ }^{42}$ B. K. Agrawal, V. Singh, A. Pathak, and R. Srivastava, Phys. Rev. B 75, 195421 (2007).

${ }^{43}$ M. M. Qazilbash, A. A. Schafgans, K. S. Burch, S. J. Yun, B. G. Chae, B. J. Kim, H. T. Kim, and D. N. Basov, Phys. Rev. B 77, 115121 (2008).

${ }^{44}$ F. Costanzo, P. L. Silvestrelli, and F. Ancilotto, J. Chem. Theory Comput. 8, 1288 (2012).

${ }^{45}$ P. L. Silvestrelli and A. Ambrosetti, J. Chem. Phys. 140, 124107 (2014).

${ }^{46}$ W. J. Fan, R. Q. Zhang, B. K. Teo, B. Aradi, and T. Frauenheim, Appl. Phys. Lett. 95, 95 (2009).

${ }^{47}$ S. S. Han and H. M. Lee, Carbon 42, 2169 (2004).

${ }^{48}$ Z. K. Horastani, S. J. Hashemifar, S. M. Sayedi, and M. H. Sheikhi, Int. J. Hydrogen Energy 38, 13680 (2013).

${ }^{49}$ S. Peng and K. Cho, Nano Lett. 3, 513 (2003). 
${ }^{50}$ T. Werder, J. H. Walther, R. L. Jaffe, T. Halicioglu, and P. Koumoutsakos, J. Phys. Chem. B 107, 1345 (2003).

${ }^{51}$ F. Leroy, S. Liu, and J. Zhang, J. Phys. Chem. C 119, 28470 (2015).

${ }^{52}$ G. Kresse and J. Hafner, Phys. Rev. B 47, 558 (1994).

${ }^{53}$ G. Kresse and J. Hafner, Phys. Rev. B 49, 14251 (1994).

${ }^{54} \mathrm{G}$. Kresse and J. Furthmüller, Comput. Mater. Sci. 6, 15 (1996).

${ }^{55}$ G. Kresse and J. Furthmüller, Phys. Rev. B 54, 11169 (1996).

${ }^{56}$ P. E. Blöchl, Phys. Rev. B 50, 17953 (1994).

${ }^{57}$ G. Kresse, Phys. Rev. B 59, 1758 (1999).

${ }^{58}$ A. D. Becke, J. Chem. Phys. 140, 18A301 (2014).

${ }^{59}$ H. S. Yu, S. L. Li, and D. G. Truhlar, J. Chem. Phys. 145, 130901 (2016).

${ }^{60}$ K. Burke, J. Chem. Phys. 136, 150901 (2012).

${ }^{61}$ J. P. Perdew and A. Zunger, Phys. Rev. B 23, 5048 (1981).

${ }^{62}$ J. P. Perdew, K. Burke, and M. Ernzerhof, Phys. Rev. Lett. 77, 3865 (1996).

${ }^{63}$ S. Grimme, J. Comput. Chem. 27, 1787 (2006).

${ }^{64}$ S. Grimme, J. Antony, S. Ehrlich, and H. Krieg, J. Chem. Phys. 132, 154104 (2010).

${ }^{65}$ S. Grimme, S. Ehrlich, and L. Goerigk, J. Comput. Chem. 32, 1456 (2011).

${ }^{66}$ A. Tkatchenko and M. Scheffler, Phys. Rev. Lett. 102, 73005 (2009).

${ }^{67}$ A. Tkatchenko, R. A. Distasio, R. Car, and M. Scheffler, Phys. Rev. Lett. 108, 236402 (2012).

${ }^{68}$ T. Bučko, S. Lebègue, J. Hafner, and J. G. Ángyán, Phys. Rev. B 87, 064110 (2013).

${ }^{69}$ A. Ambrosetti, A. M. Reilly, R. A. Distasio, and A. Tkatchenko, J. Chem. Phys. 140, 18A508 (2014).

${ }^{70}$ T. Bučko, S. Lebègue, T. Gould, and J. G. Ángyán, J. Phys.: Condens. Matter 28, 045201 (2016).

${ }^{71}$ M. Dion, H. Rydberg, E. Schröder, D. C. Langreth, and B. I. Lundqvist, Phys. Rev. Lett. 92, 246401 (2004).

${ }^{72}$ Y. Zhang and W. Yang, Phys. Rev. Lett. 80, 890 (1998).

${ }^{73}$ J. Klimeš, D. R. Bowler, and A. Michaelides, J. Phys.: Condens. Matter 22, 022201 (2010).

${ }^{74}$ A. D. Becke, Phys. Rev. A 38, 3098 (1988).

${ }^{75}$ J. Klimeš, D. R. Bowler, and A. Michaelides, Phys. Rev. B 83, 195131 (2011).

${ }^{76}$ A. D. Becke, J. Chem. Phys. 84, 4524 (1986).

${ }^{77}$ K. Lee, É. D. Murray, L. Kong, B. I. Lundqvist, and D. C. Langreth, Phys. Rev. B 82, 81101 (2010).

${ }^{78}$ I. Hamada, Phys. Rev. B 89, 121103(R) (2014).

${ }^{79}$ A. D. Becke and E. R. Johnson, J. Chem. Phys. 123, 154101 (2005).

${ }^{80}$ E. R. Johnson and A. D. Becke, J. Chem. Phys. 124, 174104 (2006)

${ }^{81}$ A. D. Becke and E. R. Johnson, J. Chem. Phys. 122, 154104 (2005).

${ }^{82}$ O. A. Vydrov and T. Van Voorhis, J. Chem. Phys. 133, 244103 (2010).

${ }^{83}$ R. Sabatini, T. Gorni, and S. De Gironcoli, Phys. Rev. B 87, 041108 (2013).

${ }^{84}$ P. Giannozzi, S. Baroni, N. Bonini, M. Calandra, R. Car, C. Cavazzoni, D. Ceresoli, G. L. Chiarotti, M. Cococcioni, I. Dabo, A. Dal Corso, S. de Gironcoli, S. Fabris, G. Fratesi, R. Gebauer, U. Gerstmann, C. Gougoussis, A. Kokalj, M. Lazzeri, L. Martin-Samos, N. Marzari, F. Mauri, R. Mazzarello, S. Paolini, A. Pasquarello, L. Paulatto, C. Sbraccia, S. Scandolo, G. Sclauzero, A. P. Seitsonen, A. Smogunov, P. Umari, and R. M. Wentzcovitch, J. Phys.: Condens. Matter 21, 395502 (2009).

${ }^{85}$ J. Sun, A. Ruzsinszky, and J. Perdew, Phys. Rev. Lett. 115, 036402 (2015). ${ }^{86}$ Y. Baskin and L. Meyer, Phys. Rev. 100, 544 (1955).

${ }^{87}$ D. Sung, S. Hong, Y.-H. Kim, N. Park, S. Kim, S. L. Maeng, and K.-C. Kim, Appl. Phys. Lett. 89, 243110 (2006).

${ }^{88}$ S. Plimpton, J. Comput. Phys. 117, 1 (1995).

${ }^{89}$ M. W. Mahoney and W. L. Jorgensen, J. Chem. Phys. 115, 10758 (2001).

${ }^{90}$ H. J. C. Berendsen, J. R. Grigera, and T. P. Straatsma, J. Phys. Chem. 91, 6269 (1987).

${ }^{91}$ J. H. Los and A. Fasolino, Phys. Rev. B 68, 024107 (2003).
${ }^{92}$ G. Pérez-Hernández and B. Schmidt, Phys. Chem. Chem. Phys. 15, 4995 (2013).

${ }^{93}$ R. J. Needs, M. D. Towler, N. D. Drummond, and P. L. Ríos, Casino version 2.13, 2010.

${ }^{94}$ Y. S. Al-Hamdani, M. Ma, D. Alfè, O. A. von Lilienfeld, and A. Michaelides, J. Chem. Phys. 142, 181101 (2015).

${ }^{95}$ Y. S. Al-Hamdani, D. Alfè, O. A. von Lilienfeld, and A. Michaelides, J. Chem. Phys. 141, 18C530 (2014).

${ }^{96}$ J. R. Trail and R. J. Needs, J. Chem. Phys. 122, 174109 (2005).

${ }^{97}$ J. R. Trail and R. J. Needs, J. Chem. Phys. 122, 014112 (2005).

${ }^{98}$ D. Alfè and M. J. Gillan, Phys. Rev. B 70, 161101 (2004).

${ }^{99}$ L. Mitas, E. L. Shirley, and D. M. Ceperley, J. Chem. Phys. 95, 3467 (1991).

${ }^{100}$ A. Zen, S. Sorella, M. J. Gillan, A. Michaelides, and D. Alfè, Phys. Rev. B 93, 241118(R) (2016).

${ }^{101}$ A. C. Dillon, K. M. Jones, T. A. Bekkedahl, C. H. Kiang, D. S. Bethune, and M. J. Heben, Nature 386, 377 (1997).

${ }^{102}$ M. Shiraishi, T. Takenobu, and M. Ata, Chem. Phys. Lett. 367, 633 (2003).

${ }^{103}$ F. Lamari Darkrim, P. Malbrunot, and G. P. Tartaglia, Int. J. Hydrogen Energy 27, 193 (2002).

${ }^{104}$ M. Hirscher, M. Becher, M. Haluska, U. Dettlaff-Weglikowska, A. Quintel, G. Duesberg, Y.-M. Choi, P. Downes, M. Hulman, S. Roth, I. Stepanek, and P. Bernier, Appl. Phys. A: Mater. Sci. Process. 72, 129 (2001).

${ }^{105}$ M. Hirscher, M. Becher, M. Haluska, F. Von Zeppelin, X. Chen, U. DettlaffWeglikowska, and S. Roth, J. Alloys Compd. 356-357, 433 (2003).

${ }^{106}$ B. Panella, M. Hirscher, and S. Roth, Carbon 43, 2209 (2005).

${ }^{107}$ H. M. Cheng, Q. H. Yang, and C. Liu, Carbon 39, 1447 (2001).

${ }^{108}$ A. Tkatchenko, A. Ambrosetti, and R. A. Distasio, J. Chem. Phys. 138, 074106 (2013).

${ }^{109}$ J. G. Brandenburg, J. E. Bates, J. Sun, and J. P. Perdew, Phys. Rev. B 94, 115144 (2016).

${ }^{110}$ M. J. Gillan, D. Alfè, and A. Michaelides, J. Chem. Phys. 144, 130901 (2016).

${ }^{111}$ A. J. Williamson, J. C. Grossman, R. Q. Hood, A. Puzder, and G. Galli, Phys. Rev. Lett. 89, 196803 (2002).

${ }^{112}$ Y. Li, D. Lu, H.-V. Nguyen, and G. Galli, J. Phys. Chem. A 114, 1944 (2010).

${ }^{113}$ J. Klimeš and A. Michaelides, J. Chem. Phys. 137, 120901 (2012).

${ }^{114}$ S. Grimme, A. Hansen, J. G. Brandenburg, and C. Bannwarth, Chem. Rev. 116, 5105 (2016).

${ }^{115}$ K. Berland, V. R. Cooper, K. Lee, E. Schröder, T. Thonhauser, P. Hyldgaard, and B. I. Lundqvist, Rep. Prog. Phys. 78, 66501 (2015).

${ }^{116}$ C. Adamo and V. Barone, J. Chem. Phys. 110, 6158 (1999).

${ }^{117}$ J. P. Perdew, M. Ernzerhof, and K. Burke, J. Chem. Phys. 105, 9982 (1996).

${ }^{118}$ S. Grimme, Wiley Interdiscip. Rev.: Comput. Mol. Sci. 1, 211 (2011).

${ }^{119}$ P. Ganesh, J. Kim, C. Park, M. Yoon, F. A. Reboredo, and P. R. C. Kent, J. Chem. Theory Comput. 10, 5318 (2014).

${ }^{120} \mathrm{~J}$. Klimeš, "Towards an accurate theoretical description of surface processes," Ph.D. thesis, UCL, University College London, 2011.

${ }^{121}$ I. Hamada, Phys. Rev. B 86, 195436 (2012).

${ }^{122}$ V. G. Ruiz, W. Liu, E. Zojer, M. Scheffler, and A. Tkatchenko, Phys. Rev. Lett. 108, 146103 (2012).

${ }^{123}$ G. Graziano, J. Klimeš, F. Fernandez-Alonso, and A. Michaelides, J. Phys.: Condens. Matter 24, 424216 (2012).

${ }^{124}$ M. J. Deible, M. Kessler, K. E. Gasperich, and K. D. Jordan, J. Chem. Phys. 143, 084116 (2015).

${ }^{125}$ A. J. Cohen, W. Y. P. Mori-Sánchez, P. Mori-Sánchez, and W. Yang, Science 321, 792 (2008).

${ }^{126}$ H. S. Yu, X. He, S. L. Li, and D. G. Truhlar, Chem. Sci. 7, 5032 (2016).

${ }^{127}$ A. White and J. F. Dobson, Phys. Rev. B 77, 75436 (2008).

${ }^{128}$ A. J. Misquitta, J. Spencer, A. J. Stone, and A. Alavi, Phys. Rev. B 82, 75312 (2010). 